\title{
ReaR
}

\section{Gravedad del síndrome de apnea-hipopnea obstructiva del sueño, raza, edad y patrón de crecimiento como indicadores de complicaciones respiratorias tras adenoamigdalectomía, en paciente pediátrico.}

Ferreras Vega R, Rubio Pascual P, Méndez Marín MD.

\section{H.U. 12 de Octubre, Madrid.}

\section{Resumen}

En la mayor parte de los casos de SAHOS pediátrico, el tratamiento de elección es la amigdalectomía con adenoidectomía. En la consulta preanestésica, la evaluación de la gravedad del SAHOS, edad, crecimiento y raza pueden ser relevantes si se quiere optar por un circuito ambulatorio. El objetivo de este artículo es la revisión de los factores de riesgo para sufrir complicaciones respiratorias, en el perioperatorio de amigdalectomía y adenoamigdalectomía.

El SAHOS grave aumenta la incidencia de eventos adversos, siendo la polisomnografía es la prueba de elección para su evaluación. Sin embargo, no parece claramente asociada con complicaciones mayores o sangrado, por lo que la experiencia del equipo y los recursos disponibles, pueden hacer posible una CMA para AA.

\section{Introducción}

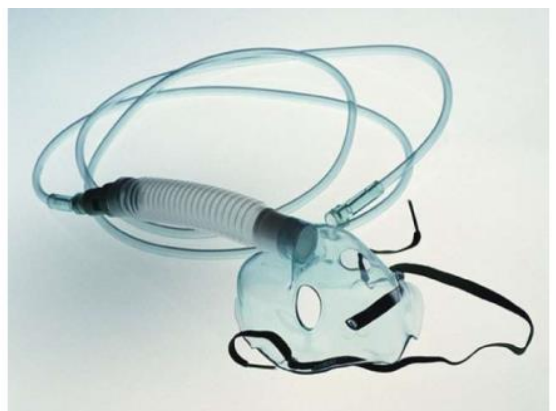

En la mayor parte de los casos de SAHOS pediátrico, el tratamiento de elección es la amigdalectomía con adenoidectomía. En la consulta preanestésica, la evaluación de la gravedad del SAHOS, edad, crecimiento y raza pueden ser relevantes si se quiere optar por un circuito ambulatorio. El objetivo de este artículo es la revisión de los factores de riesgo para sufrir complicaciones respiratorias, en el perioperatorio de

\section{amigdalectomía}

adenoamigdalectomía.

El SAHOS grave aumenta la incidencia de eventos adversos, siendo la polisomnografía es la prueba de elección para su evaluación. Sin embargo, no parece claramente asociada con complicaciones mayores $\mathrm{O}$ sangrado, por lo que la experiencia del equipo y los recursos disponibles, pueden hacer posible una CMA para AA.

El tratamiento que suele aplicarse en la mayor parte de los casos de Síndrome de apnea-hipopnea obstructiva del sueño (SAHOS) pediátricos, es la amigdalectomía con adenoidectomía. Si para su realización se opta por un circuito ambulatorio, la evaluación preanestésica es fundamental.

El objetivo de esta revisión bibliográfica es relacionar 
individualmente

identificables en la consulta preanestésica (gravedad del SAHOS, raza, edad y patrón de crecimiento) con el riesgo de sufrir complicaciones respiratorias, en el perioperatorio de amigdalectomía adenoamigdalectomía.

Concluimos que el SAHOS grave aumenta la incidencia de eventos respiratorios adversos. Sin embargo, no parece claramente asociada con otras complicaciones mayores, por lo que la experiencia del equipo y los recursos disponibles, pueden hacer posible una cirugía mayor ambulatoria (CMA) para adenoamigdalectomía. La ascendencia africana implica una mayor tasa de eventos respiratorios indeseables. Los niños menores de 3 años van a presentar una incidencia mayor de complicaciones independientemente de su peso. Los mayores de 3 años con menos de $18 \mathrm{~kg}$ asociarían mayor tasa de ingreso en unidades de intensivos tras adenoamigdalectomía.

\section{Introducción}

La incidencia del ronquido en la población pediátrica es muy variable, oscilando entre $3 \%$ y $35 \%$, en los niños menores de 13 años (1). Además, esta es más frecuente entre los 2 y 8 años (2). Por otra parte, la prevalencia de apnea-hipopnea obstructiva del sueño (SAHOS infantil), se estima en torno al $3.5 \%$ (1) (2).

En la mayor parte de los casos de SAHOS pediátrico, el tratamiento de elección es la amigdalectomía con adenoidectomía. Esta intervención, puede ser realizada en régimen de cirugía mayor ambulatoria (CMA). Los criterios de selección para optar por la vía ambulatoria son cada vez más amplios, con un impacto favorable en el coste económico y la satisfacción del paciente. No obstante, las complicaciones respiratorias inesperadas en CMA, pueden aparecer desde un $1.44 \%$ hasta el $8 \%$ de la población pediátrica $(3,4)$. Por lo tanto, la aplicación de criterios de selección en la consulta preanestésica es importante para la disminución de las mismas. En los pacientes ASA I y II, parámetros como la evaluación de la gravedad del SAHOS, raza, edad y patrón de crecimiento resultan relevantes en la predicción de complicaciones.

\section{Objetivo}

Realizar una revisión bibliográfica para conocer el impacto que ejercen individualmente, en el niño, factores de riesgo identificables en la consulta preanestésica (gravedad del SAHOS, raza edad y patrón de crecimiento) sobre los eventos indeseables respiratorios, tras adenoamigdalectomía.

\section{Métodos}

Se elabora una revisión bibliográfica, consultando fuentes primarias a través de la base de datos PubMed $\AA$, EMBASE ®y Uptodate. Los criterios de selección de los artículos están basados en la conexión del tema tratado utilizando los siguientes términos de búsqueda: "Adenotonsillectomy" OR "tonsillectomy" AND "complications" en combinación con términos que acotan población infantil, cirugía ambulatoria y complicaciones respiratorias: "children", "pediatric", "respiratory events", "day case surgery".

Las publicaciones seleccionadas fueron revisiones retrospectivas y metanálisis. Para la valuación de la fiabilidad se ha tomado en cuenta su factor de impacto, en función del número de veces citado en otros artículos, estableciendo un margen de un mínimo de 6 citas. 


\section{Resultados}

Se seleccionan un total de 14 publicaciones originales con un mínimo de 6 citas bibliográficas. En estos artículos la edad menor a 3 años, el SAHOS grave, el retardo en el crecimiento y la raza, son criterios que parecen asociados a aumento de eventos indeseados tras adenoamigdalectomía.

\section{Discusión}

A continuación exponemos los distintos factores de riesgo (SAHOS, la raza, la edad y el patrón de crecimiento) y su relación individual con las complicaciones respiratorias, según los datos encontrados en las publicaciones seleccionadas.

\section{SAHOS}

La gravedad del SAHOS está en relación directa con la aparición de eventos respiratorios no deseados tras adenoamigdalectomía. Para evaluar el SAHOS, tanto el ronquido como el trabajo respiratorio son predictores poco fiables. Así, siguiendo las recomendaciones de la Academia Americana de Pediatría (2.002), la polisomnografía sería la prueba que mejor predice la gravedad del SAHOS (5). No obstante, no siempre es posible disponer de ella en los niños, sobre todo en los de menor edad, dada su escasa colaboración. Así, otros métodos de estimación indirectos han cobrado importancia en la población pediátrica. Entre ellos, el uso de cuestionarios del sueño, la pulsioximetría nocturna, la endoscopia bajo anestesia y los marcadores urinarios (6), son considerados relevantes en esta valoración. Los cuestionarios del sueño son preguntas estructuras que se pueden realizar en la consulta, como el BEARS $(\mathrm{B}=$ Problemas para acostarse, $\mathrm{E}=$ Excesiva somnolencia diurna, $\mathrm{A}=$ Despertares durante la noche, $\mathrm{R}=$
Regularidad y duración del sueño, $\mathrm{S}=$ Ronquidos "snoring"). Además, son capaces de detectar otros problemas como los despertares nocturnos. La pulsioximetría nocturna cuantifica las variaciones de saturación durante las apneas, requiriéndose un índice de desaturaciones alto (más de 10) para considerarse patológica.

Los resultados del metanálisis llevado a cabo por de Luca Canto (7), concluyen que la aparición de eventos respiratorios tras adenoamigdalectomía es más frecuente en niños con SAHOS respecto a niños sin SAHOS, creciendo según aumenta la gravedad de este.

En concordancia, Kang et als (8), establecieron que a medida que la gravedad del SAHOS aumentaba, también lo hacían las complicaciones respiratorias, en el postoperatorio de la adenoamigdalectomía. Siendo, la desaturación, durante la estancia en la unidad de recuperación postanestésica (URPA), la complicación asociada más frecuente. No obstante, en sus resultados los niños que desarrollaron complicaciones respiratorias mayores (laringoespasmo, broncoespasmo, edema pulmonar, neumonía $\mathrm{o}$ intubación durante estancia en URPA), no parecía ser determinante la gravedad de SAHOS.

Por otra parte, Arambula (9), en un análisis retrospectivo de adenoamigdalectomía en niños, concluye que los niños que ingresan en intensivos tenían edades menores $\mathrm{y}$ SAHOS más graves que los niños que solo han necesitado estancia en URPA. Los trabajos de Kang y Arambula, que fueron publicados en 2.017 y 2.018 , pueden parecer discordantes indicando que la exclusión del SAHOS moderado y leve, para una adenoamigdalectomía en CMA, no es una decisión fácil. Factores relevantes para el tratamiento de estos niños serian la experiencia del 
equipo y los recursos disponibles en el centro.

\section{- Raza}

Mediante una revisión retrospectiva, Horwood et als (10) examinaron en 594 pacientes pediátricos la incidencia de eventos respiratorios mayores tras adenoamigdalectomía. Fueron considerados eventos graves la ventilación con bolsa, reintubación y necesidad de medicamentos para control respiratorio. Aparecieron con más frecuencia en $\leq 2$ años $(\mathrm{P}<.001)$, SAHOS grave $(\mathrm{P}=0.003)$ y cuando presentaban alteraciones del crecimiento $(\mathrm{P}=0.04)$. Aplicaron un análisis multivariante (edad, peso, SAHOS, otras comorbilidades) específicamente los niños de etnia afroamericana. Fueron clasificados como pertenecientes a esta etnia aquellos que tenían un rasgo o anemia facilforme. El $19 \%$ de los niños con test falciforme positivo experimentaron una complicación respiratoria mayor, comparado con el $10 \%$ con test negativo: OR: 1.89 [IC 95\%, 1.28-2.80] $(\mathrm{P}=0.01)$.

En el año 2.019, Kou et al (11) publicaron una revisión retrospectiva que incluyó 30.617 niños sometidos a amigdalectomía. Las variables, edad, género, peso, SAHOS y asma, fueron controladas. Los resultados concluyeron que los niños afroamericanos tenían mayor incidencia de laringo $y$ broncoespasmo, neumonía, edema pulmonar, intubación y ventilación mecánica que los niños de raza blanca, OR: 1.5, [IC95\%: 1.3-1.6].

\section{Edad y crecimiento}

La incidencia de eventos adversos tras amigdalectomía, en niños mayores de 3 años ASA I y II, parece baja.
El punto de corte situado en 3 años, ha sido establecido en función de los datos extraídos de diversos estudios. Statham et al, en el año 2006 (12) publicaron un análisis retrospectivo sobre 2315 pacientes menores de 6 años operados de adenoamigdalectomía. Observaron que las complicaciones postoperatorias, tienen una incidencia mayor en menores de 3 años, comparado con los de 3-5 años $(9.8 \%$ vs $4.9 \% \mathrm{p}=0.001)$. Entre las incidencias asociadas a la intervención, las más comunes son los problemas respiratorias en el posoperatorio, (OR: 1.98; 95\% IC: 1.41- 2.77). Como eventos respiratorios indeseables destacan: las desaturaciones de oxígeno por debajo del 90\% (las más frecuente), las apneas o aumento del trabajo respiratorio, precisando ventilación mecánica no invasiva (VMNI) o invasiva (VMI), atelectasias, infiltrados o edemas pulmonares, neumomediastino, neumotórax y los derrames pleurales. Es reseñable que, en este estudio, la mayoría de los pacientes menores de 3 años, no padecían comorbilidades (obesidad, asma...) que si presentaban los pacientets del grupo de 3-5 años.

Claire M. Lawlor et al (4) en el año 2.018, obtienen resultados en este sentido. Fueron estudiados 2139 pacientes menores de 6 años (1960 finalmente fueron incluidos), sometidos a amigdalectomía. El objetivo principal de este estudio fue determinar si, los niños sanos menores de 3 años, tenían más eventos indeseables postoperatorios que los pacientes entre 3 a 6 años. Secundariamente valoraron, si el peso estaba asociado con un mayor riesgo de eventos adversos. Concluyeron que, independientemente del peso, los niños menores de 3 años presentaban mayor número de complicaciones (odds ratio [OR], 1.56; 95\% CI, 1.00-2.42). No encontraron diferencias estadísticamente significativas $(\mathrm{P}=0.66)$ entre las incidencias ocurridas en niños 
menores de $10 \mathrm{~kg}$ y mayores de $10 \mathrm{~kg}$, independientemente de la edad.

En cuanto al crecimiento, la evaluación del desarrollo pondoestatural resulta también útil para decidir usar un circuito ambulatorio.

Es conocida la relación entre los trastornos del sueño secundarios a hipertrofia amigdalina y la alteración en el crecimiento del niño (13). Si evaluamos el crecimiento, este sigue un patrón escalonado y no continuo, por ello hay una gran variabilidad entre las distintas etapas de crecimiento.

Una adecuada valoración debe ser prolongada en el tiempo y no de un solo registro de peso y talla. En este sentido, Kalberg ideó un modelo matemático que corresponde a la curva de crecimiento. Si hay cambios negativos podemos encontrarnos ante el llamado fallo de medro.

Zenel (1997) aportó tres definiciones respecto al fallo de medro:

1. Un niño menor de 2 años de edad cuyo peso está por debajo de los percentiles 3 ó 5 para la edad, en más de una ocasión.

2. Un niño menor de 2 años de edad cuyo peso es inferior al $80 \%$ del peso ideal para la edad.

3. Un niño menor de 2 años de edad cuyo peso cae dos percentiles, a lo largo del tiempo, en una gráfica de crecimiento estandarizada.

Son consideradas excepciones: la estatura genética corta, retrasos crecimiento intrauterino, sobrepeso con velocidad en talla aumentada, pero ganancia ponderal disminuida, y niños constitucionalmente delgados.
El fallo de medro es un concepto que hace referencia a los niños menores de 2 años, aunque, otros autores amplían esa edad hasta los 3 años. Es vital esta diferenciación porque los pacientes entre 2-3 años son los que más dudas generan a la hora de asignarles una vía ambulatoria.

Para la evaluación en la consulta preoperatoria del niño con sospecha de fallo de medro (tabla-1) debe constar: el punto temporal dónde se empieza a detectar, los parámetros afectados y si existen $\mathrm{o}$ no factores intercurrentes (como enfermedades de base, factores sociales...). Cuando la ausencia de ganancia ponderal se produce de forma aguda ese fenómeno se conoce como "wasting" (consumido). $\mathrm{Si}$ esta situación se extiende en el tiempo conlleva atrofia (tabla-1).

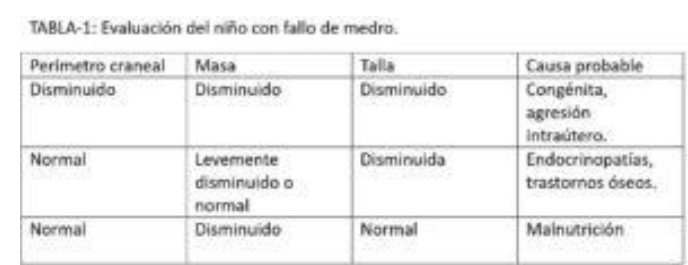

TABLA 1, fuente: documento de la sociedad española de pediatría sobre el fallo de medro. Disponible

http://www.aeped.es/sites/default/files/documen tos/9-fallo_medro.pdf

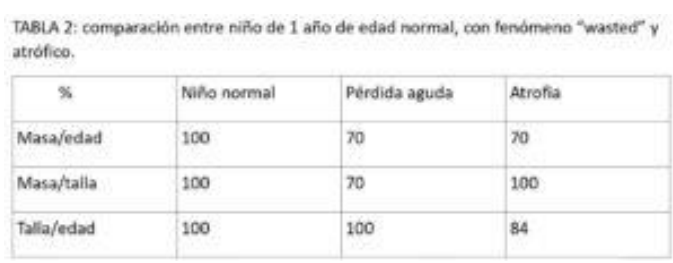

Tabla-2, fuente: Waterlow JC: Nutrition and growth. En: Protein energy malnutrition, London, 1992, Edward Arnold, 187-211.

El impacto del crecimiento tiene efecto en la aparición de complicaciones asociadas a adenoamigdalectomía.

Julien-Marsollier et al. (14), hicieron un análisis retrospectivo sobre 805 pacientes pediátricos, mayores de 3 años, en los que practicaron una amigdalectomía. 93 entraron en la 
unidad de cuidados intensivos: 68 planeados y 25 pacientes desarrollaron un fallo respiratorio postoperatorio imprevisto. Con estos datos, establecieron que los niños con menos $18 \mathrm{~kg}$, asociaban mayor tasa de eventos postoperatorios (ingreso en intensivos y necesidad de apoyo ventilatorio), ROC 0.9 [IC 95\%: 0.85-0.95]. En este trabajo el sobrepeso ( $>95 \%$ del percentil del IMC), al igual que el SAHOS grave fueron criterios de exclusión para CMA (tabla 3). El resto de factores de riesgo no excluidos son homogéneos en los distintos grupos. Estos resultados no concuerdan con el trabajo previamente citado de Lawlo et al (4), pudiendo entrar en conflicto los criterios edad y peso. No obstante, encontramos diferencias metodológicas significativas al comparar ambos estudios $(4,14)$. Estas pueden ayudarnos a entender las conclusiones, a priori dispares, de los dos investigadores.

En lo referente a los criterios de inclusión, observamos variabilidad entre los dos trabajos. Lawlo centra sus investigaciones en la edad, por lo que el peso del paciente pasa a un lugar secundario, de los 1960 totales solo 33 pacientes $(1,68 \%)$ presentaban una masa menor a $10 \mathrm{~kg}$. La $\mathrm{p}$ de 0.66 , al comparar complicaciones entre menores de $10 \mathrm{~kg}$ y mayores de $10 \mathrm{~kg}$, no encuentra diferencias estadísticamente significativas, pero puede ser debido a la disparidad de $n$.

En cuanto a criterios de exclusión (tabla 3) de Julien-Marsollier, en lo referente la edad menor a 2 años y el sobrepeso, también juegan un papel clave a la hora de comparar ambos trabajos.

Podemos observar que los dos autores abren una línea a seguir, aunque cada uno se focaliza en puntos clave (edad y peso), pero futuros estudios comparativos podrían arrojar más luz a estas cuestiones.

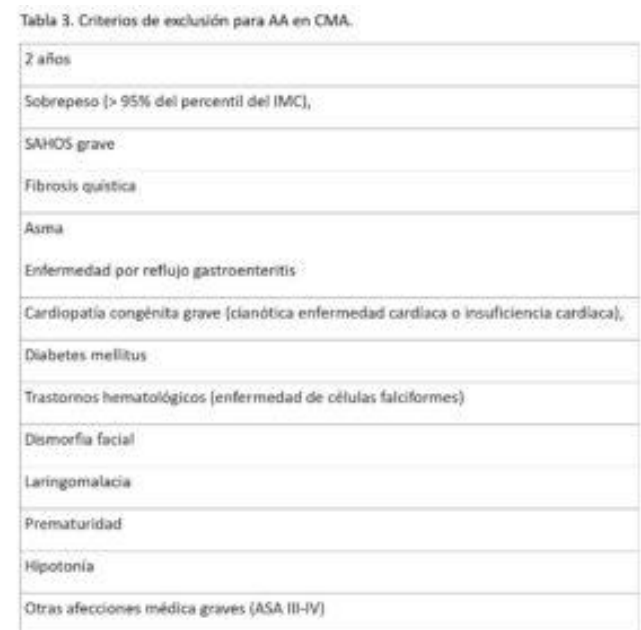

Tabla-3, fuente: (14) Julien-Marsollier F, Salis P, Abdat R, Diallo T, Van Den Abbelle T, Dahmani S. Predictive factors of early postoperative respiratory complications after tonsillectomy in children with unidentified risks for this complication. Anaesth Crit Care Pain Med. 2018;37(5):439-445.

\section{Conclusiones}

Una de las indicaciones de la adenoamigdalectomía en el niño son los trastornos del sueño. Es ampliamente usada la vía ambulatoria para la realización de esta cirugía y los criterios cada vez son más amplios. Con el fin de evitar complicaciones imprevistas es necesaria una cuidadosa evaluación en la consulta preoperatoria. La polisomnografía es el patrón oro para la evaluación de la gravedad del SAHOS pero no siempre es posible realizarla. El SAHOS grave aumenta la incidencia de eventos respiratorios adversos. Sin embargo, no parece claramente asociada con otras complicaciones mayores. Por ello, la experiencia del equipo y los recursos disponibles, pueden hacer posible una CMA para adenoamigdalectomía. La ascendencia africana, evaluada con test facilforme positivo, implica una mayor tasa de eventos respiratorios indeseables tras amigdalectomía, por lo que hay que tener en cuenta también este factor, a la hora de crear una lista quirúrgica ambulatoria. La edad, la evaluación del crecimiento y la raza pueden ser de ayuda para tomar esta decisión, sobre 
todo en pacientes entre 2-4 años. Los niños menores de 3 años van a presentar una incidencia mayor de complicaciones independientemente de su peso, por lo que la mayoría de grupos evita el alta precoz, en este intervalo de edad. La evaluación del crecimiento debe basarse en un patrón temporal, los pacientes con alteración en el desarrollo ponderal y SAHOS, representan un factor de riesgo respiratorio. Así, los mayores de 3 años con menos de $18 \mathrm{~kg}$ asociarían mayor tasa de ingreso en unidades de intensivos tras adenoamigdalectomía.

\section{Bibliografía}

1. Castronovo V, Zucconi M, Nosetti L, Marazzini C, Hensley M, Veglia F, Nespoli L, Ferini-Strambi L. Prevalence of habitual snoring and sleep-disordered breathing in preschoolaged children in an Italian community. Journal Pediatr. 2003; 142(4):377-82. (ㄴML)

2. Schlaud M, Urschitz MS, Urschitz-Duprat PM, Poets CF. The German study on sleepdisordered breathing in primary school children: epidemiological approach, representativeness of study sample, and preliminary screening results. Paediatr Perinat Epidemiol.2004; 18(6):431-40. (PubMed)

3. Raman VT, Jatana KR, Elmaraghy CA, Tobias JD. Guidelines to decrease unanticipated hospital admission following adenotonsillectomy in the pediatric population. Int J Pediatr Otorhinolaryngol. 2014;78(1):1922 ( $\underline{\text { HTML) }}$

4. Lawlor CM, Riley CA, Carter JM Rodriguez KH. Association Between Age and Weight as Risk Factors for Complication After Tonsillectomy in Healthy Children.JAMA Otolaryngol Head Neck Surg. 2018;144(5):399405. (

5. Garg RK, Afifi AM, Garland CB, Sanchez R, Mount DL Pediatric Obstructive Sleep Apnea: Consensus, Controversy, and Craniofacial Considerations. Plast Reconstr Surg. 2017; 140(5):987-997 (․ubMed)

6. McGrath B, Lerman J. Pediatric sleepdisordered breathing: an update on diagnostic testing. Curr Opiln Anaesthesiol. 2017;30(3):357-361. (PubMed)

7.De Luca Canto G, Pachêco-Pereira C, Aydinoz S, Bhattacharjee R, Tan HL, Kheirandish-Gozal L, Flores-Mir C, Gozal D. Adenotonsillectomy Complications: A Metaanalysis. Pediatrics. 2015;136(4):702-18. (PMC)

8. Kang KT ,Chang IS,Tseng CC Weng WC, Hsiao TY, Lee PL Hsu WC. Impacts of disease severity on postoperative complications in children with sleep-disordered breathing.Laryngoscope. 2017;127(11):26462652. ( $\underline{\text { SAGE}})$

9. Arambula AM, Xie DX Whigham AS.Respiratory events after adenotonsillectomy requiring escalated admission status in children with obstructive sleep apnea.Int J Pediatr Otorhinolaryngol. 2018 Apr;107:31-36. (

10. Horwood L, Nguyen LH, Brown K, Paci P, Constantin E. African American ethnicity as a risk factor for respiratory complications following adenotonsillectomy. AMA Otolaryngol Head Neck Surg. 2013;139(2):147152 ( $\underline{\text { JAMA })}$

11. Kou YF, Sakai M, Shah GB, Mitchell RB, Johnson RF. Postoperative respiratory complications and racial disparities following inpatient pediatric tonsillectomy: A crosssectional study. Laryngoscope. 2019; 129(4):995-1000.

12. Statham MM, Elluru RG, Buncher R,Kalra M. Adenotonsillectomy for obstructive sleep apnea syndrome in young children: prevalence of pulmonary complications.Arch Otolaryngol Head Neck Surg. 2006;132(5):476-80. (

13. Bonuck K,Parikh S,Bassila M. Growth failure and sleep disordered breathing: a review of the literature. Int J Pediatr Otorhinolaryngol. 2006;70(5):769-78 (PubMed)

14. Julien-Marsollier F, Salis P, Abdat R, Diallo T, Van Den Abbelle T, Dahmani S. Predictive factors of early postoperative respiratory complications after tonsillectomy in children with unidentified risks for this complication. Anaesth Crit Care Pain Med. 2018;37(5):439445. (PubMed) 
Lectura

recomendada:https://www.ncbi.nlm.nih.gov/p $\underline{\text { mc/articles/PMC5330066/ }}$
Correspondencia al autor

Rubén Ferreras Vega

rubenferrerasvega@hotmail.com

FEA adjunto al servicio de anestesia pediátrica H.U. 12 de Octubre, Madrid

Aceptado para el blog en enero de 2021 